\title{
High-performance Multiplexer-based Logic Synthesis Using Pass-transistor Logic
}

\author{
SHEN-FU HSIAO*, JIA-SIANG YEH and DA-YEN CHEN \\ Department of Computer Science and Engineering, National Sun Yat-Sen University, Kaohsiung, Taiwan
}

(Received 13 February 2001; Revised 2 March 2001)

\begin{abstract}
An automatic logic/circuit synthesizer is developed which takes several Boolean functions as input and generates netlist output with basic composing cells from the pass-transistor cell library containing only two types of cells: 2-to-1 multiplexers and inverters. The synthesis procedure first constructs efficient binary decision diagrams (BDDs) for these Boolean functions considering both multi-function sharing and minimum width. Each node in the BDD trees is realized by using a 2-to-1 multiplexer (MUX) of proper driving capability designed pass-transistor logic. The inverters are then inserted all along the MUX paths in order to improve the speed performance and to alleviate the voltage-drop problem. Several methods are proposed to reduce the critical path delay in the multiplexer-chains for generation of faster circuits. Compared to the recently proposed pass-transistor-based top-down design, our synthesizer has better speed and area performance due to the reduced number of cascaded inverters.
\end{abstract}

Keywords: Logic synthesis; Pass-transistor logic; Standard cell library; Top-down design; Binary decision diagram; VLSI

\section{INTRODUCTION}

Top-down design using high-level synthesis tools (such as Synopsys design compiler) with conventional CMOS standard cell library is one of the current mainstreams in the cell-based design methodology. However, the commercially available CMOS cell library usually contains hundreds of different cells and, thus, takes a lot of time to migrate to a new process technology, since the layout of all the cells needs to be redesigned for a new target technology. Recently, pass-transistor logic circuits that go beyond the conventional complementary CMOS have received much attention [1-4]. Among them are CPL (Complementary Pass-transistor Logic) and DPL (Double Pass-transistor Logic). One problem with the CPL or DPL circuits is the requirement of both noninverting and inverting signals, which leads to a larger wiring area. Hence, Yano et al. [5] proposed a new topdown logic design methodology based on CPL-like circuits, called LEAP (Lean Integration with Passtransistors), which uses only the non-inverting output of the original CPL multiplexer circuit appended by a p-latch inverter. Contrary to the CMOS cell library that usually contains hundreds of different cells, the above LEAP cell library consists of only a small number of pass-transistor cells, including three types of so-called Y-cells and an inverter. Thus, it is much easier to update the cell library.

The conventional CMOS cell library contains a wide variety of logic gates with different driving capability and the current synthesis tool such as Synopsys design analyzer usually exploits the features of these logic gates during the logic optimization process. However, the LEAP cell library contains only multiplexer-like cells. Most commercial logic synthesizers cannot be efficiently applied to the generation of the pass-transistor logic circuits. Thus, in Ref. [5], the authors have developed their own logic/circuit synthesizer called "circuit inventor" which fully utilizes the features of the LEAP cells to achieve better synthesis results. However, LEAP circuits might cause unnecessary cascaded inverters that increase the delay as well the total area of the synthesized circuits. In this paper, we will design a new logic/circuit synthesis tool based on a new pass-transistor cell library consisting of only two types of cells: 2-to-1 multiplexers (with two nMOS transistors only) and inverters (with or without platch). The synthesizer first generates their corresponding binary decision diagrams (BDDs) for several Boolean functions, possibly shared for hardware minimization. Afterwards, each node is implemented by a 2-to-1 multiplexer realized with two nMOS transistors.

*Corresponding author. E-mail: sfhsiao@cie.nsysu.edu.tw 

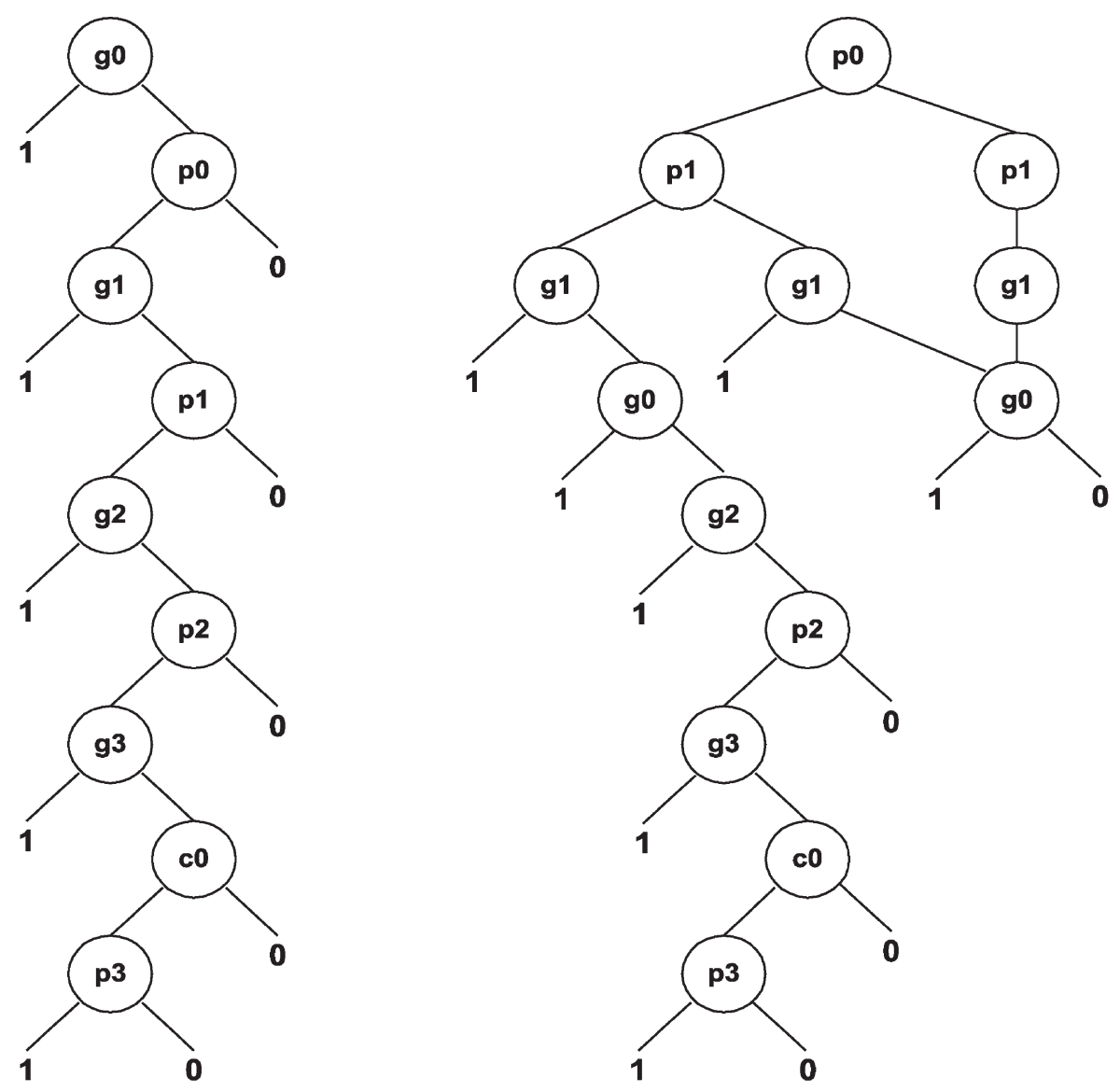

FIGURE 1 Two BDD trees generated from Shannon's expansion with respect to two different variable orders, one in order of $\left\{g_{0}, p_{0}, g_{1}, p_{1}, g_{2}, p_{2}, g_{3}, c_{0}, p_{3}\right\}$ while the other in order of $\left\{p_{0}, p_{1}, g_{1}, g_{0}, g_{2}, p_{2}, g_{3}, c_{0}, p_{3}\right\}$.

(a)
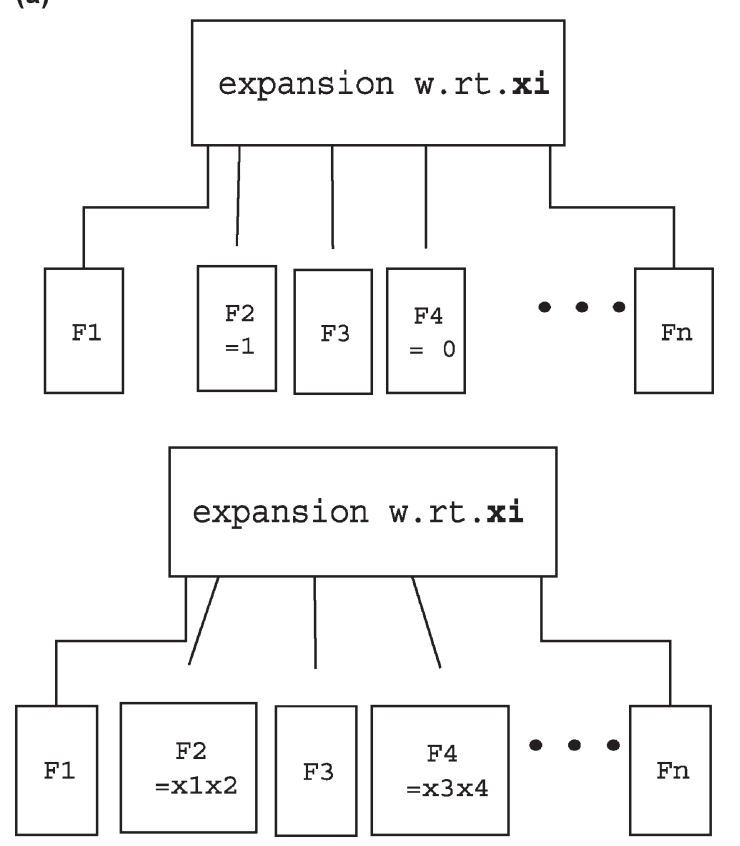

(b)
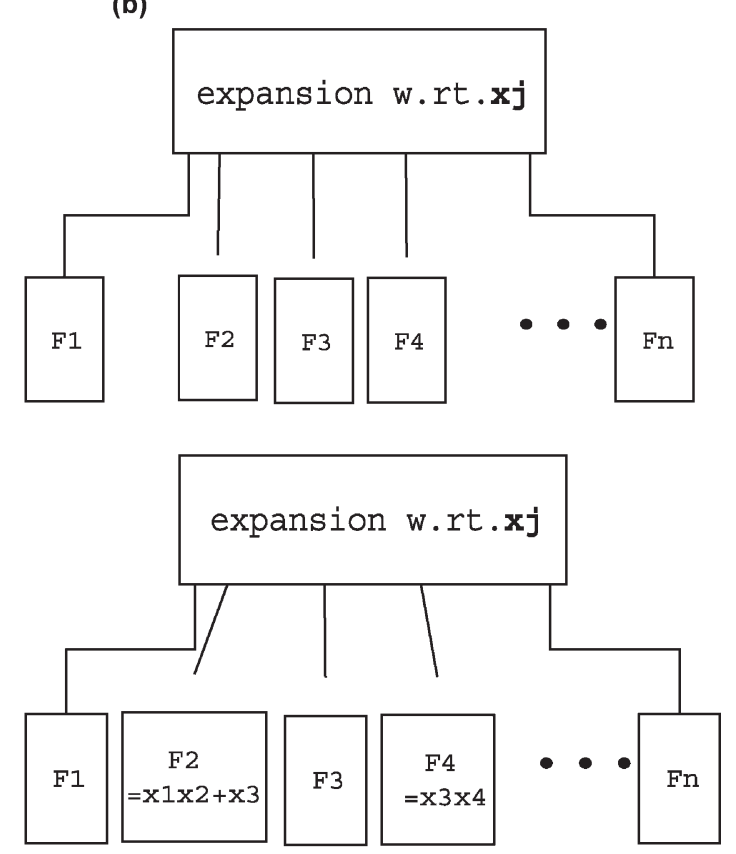

FIGURE 2 Order selection based on (a) the number of leaves (0's or 1's), and (b) the complexity of the sub-functions, when the width is the same. 


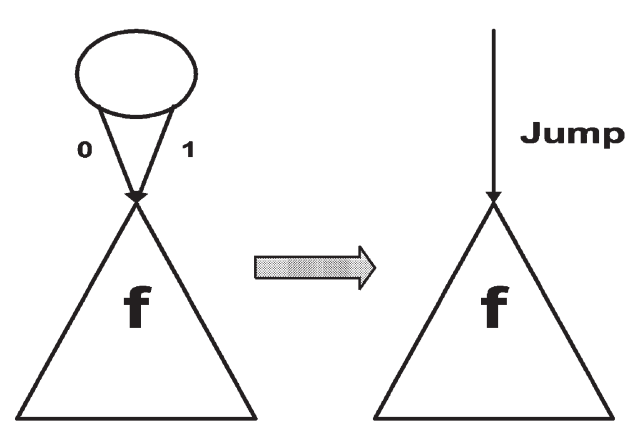

(a)

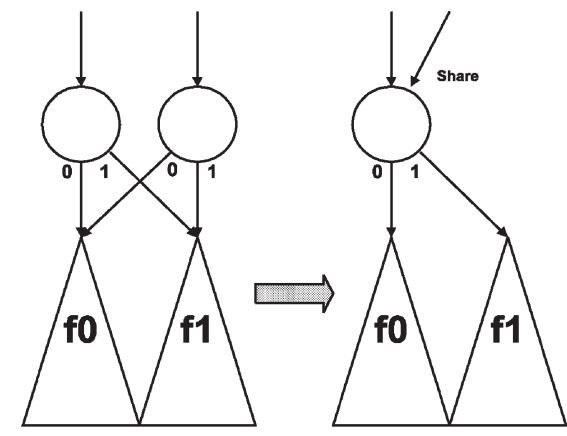

(b)

FIGURE 3 (a) Node elimination and (b) node sharing.

Then, inverters or buffers are inserted along the multiplexer chains in order to provide enough driving capability and to solve the problems of voltage drop across nMOS pass transistors. We also propose several methods to improve the speed performance of the synthesized circuits by using higher order multiplexers and by parallelizing the critical multiplexer chains.

\section{CONSTRUCTION OF BDD TREES}

Any Boolean equation can be expressed as a BDD tree by using Shannon's expansion according to a specific order of the Boolean variables [6]. The construction of BDD is affected by the following factors.

\section{Order of Boolean Variables}

It is well known that different orders of variable leads to different BDD trees. For example, Fig. 1 shows two expansions with respect to different variable orders for the 4-bit carry-look ahead addition where $p_{i}$ and $g_{i}$ are, respectively, the propagate and generate signals of the $i$-th bit. In order to reduce the number of nodes within reasonable computation time, we adopt a greedy algorithm called minimum width method which calculates the number of sub-functions each time a Boolean function is expanded with respect to a variable, and selects the expansion with the fewest number of sub-functions, i.e. the smallest width. The reason is that when the number of subfunctions for further expansion is smaller, it is more likely to have smaller width after the entire expansion process. When the number of sub-functions is the same, our proposed selection rule is to choose the expansion that leads to more leaves of 0's or 1's. For example, as shown in Fig. 2a, expansion with respect to $x_{j}$ has higher priority than that with respect to $x_{i}$ since the former has more leaves of 0 's or 1 's. If both the number of sub-functions and the number of 0's or 1's are all the same, we select the expansion which leads to less complicated sub-functions, as shown in Fig. 2b. Again, the reason for such choice is based on the consideration of generating BDD tree with width as small as possible and less complicated subfunctions that have better chance of generating more simple BDD trees.

\section{Node Elimination and Node Sharing}

Since the number of nodes in a BDD tree corresponds to the number of 2-to-1 multiplexers needed in practical implementation, fewer nodes lead to less hardware cost. We observe that when the 0 - and 1-edge of a node point to the same sub-function, we may eliminate this node, as shown in Fig. 3a. When the 0- and 1-edge of two or more nodes points the same sub-function, these nodes can be degenerated into a single node, as shown in Fig. 3 b.

\section{Multi-function Sharing}

Sometimes, we want to create the BDD trees for more than one Boolean function. It is possible to share part of these BDD trees if there exists the same sub-functions or the complement of the sub-functions during the expansion process. Figure 4 shows the sharing of the same subfunctions in the generation of two BDD trees for two different Boolean functions where F1 is a sub-function of F2.

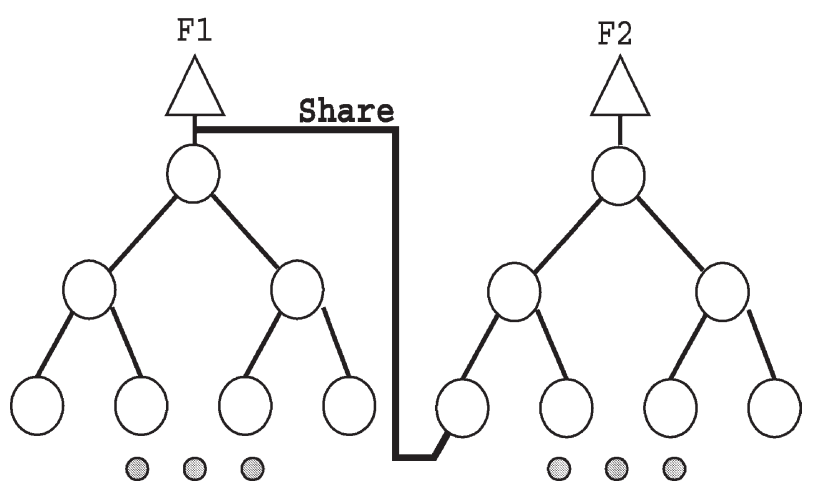

FIGURE 4 Multi-function sharing in two different BDD trees. 


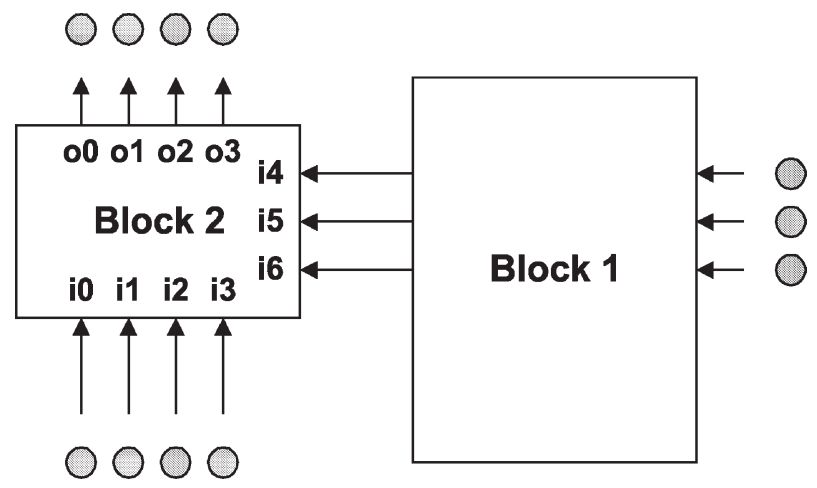

FIGURE 5 Input signals $i_{4}, i_{5}, i_{6}$ (with larger signal arriving time) of circuit block 2 may have higher priority (specified by users) than $i_{0}, i_{1}, i_{2}$, $i_{3}$ (from the primary inputs) during the expansion, in order to generate faster circuits.

\section{User-defined Variable Priority}

As mentioned before, the selection of variable order for BDD generation is usually based on the criterion of minimizing the width and the number of nodes. However, in practice, designers may define their own variable order according to realistic implementation situation. For example, if not all the input variables of a Boolean equation arrive at the same time, one might give the slowest input variable the highest priority (at the top of the BDD tree) during Shannon's expansion so that the delay from this primary input to the output (at the root of the BDD tree) passes fewest multiplexers. This option is particularly important when a designer wants to balance the delay in a large system, such as the parallel array multiplier. For example, in Fig. 5 , the input signals $i_{4}, i_{5}, i_{6}$ of circuit block 2 comes from another block 1 and, thus, usually have larger delay than other input signals $i_{0}, i_{1}, i_{2}$, $i_{3}$ that are assumed to be the primary inputs. Thus, users may decide to generate a BDD tree with input signals $i_{4}, i_{5}$, $i_{6}$ at the upper levels of the tree in order to balance the delay and to generate faster circuits.

\section{IMPLEMENTATION USING PASS-TRANSISTOR LOGIC CELL LIBRARY}

Once the Boolean functions are represented by BDD trees, we can easily realize the logic functions by replacing each node in the BDD trees with a 2-to-1 multiplexer designed using pass-transistor logic, such as CPL or DPL. The output of the CPL-like multiplexer, as shown in Fig. 6a, can only be connected to the source/drain input of another MOS transistor while the DPL-like multiplexer, as shown in Fig. 6b, can be connected to any terminal of an MOS transistor. The conventional CMOS design of a 2-to-1 multiplexer cell requires more hardware area and, thus, is not adopted in our synthesizer. Due to the voltage drop through nMOS pass-transistors, it is also necessary to add inverters or buffers throughout the BDD trees. The p-latch inverter shown in Fig. $6 \mathrm{c}$ is inserted into the BDD tree whenever the number of cascaded CPL-like 2-to-1 multiplexers is more than a user-specified number

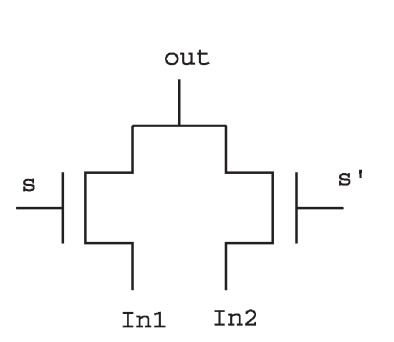

(a)

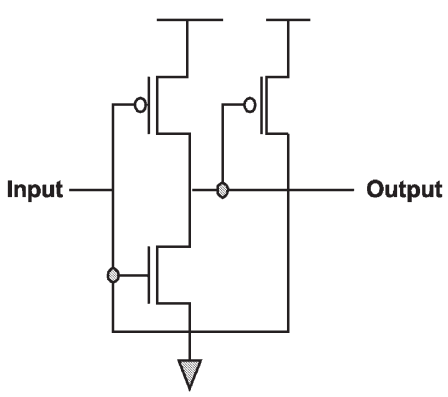

(c)

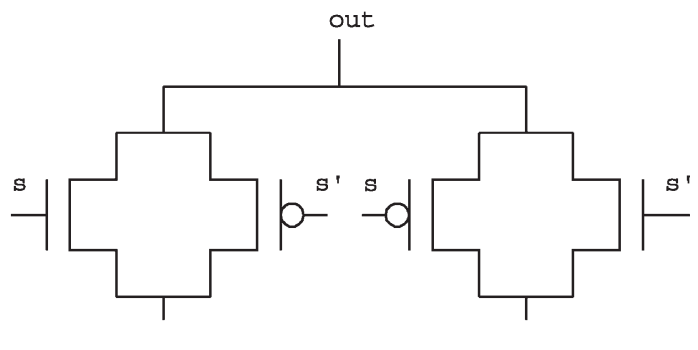

(b)

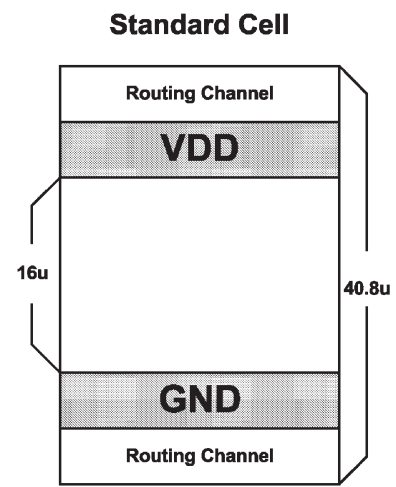

(d)

FIGURE 6 2-to-1 multiplexer realized using (a) nMOS pass transistors only, (b) using CMOS transmission gates, (c) p-latch inverter, (d) aspect ratio of the layout in the pass-transistor-based cell library. 
F1

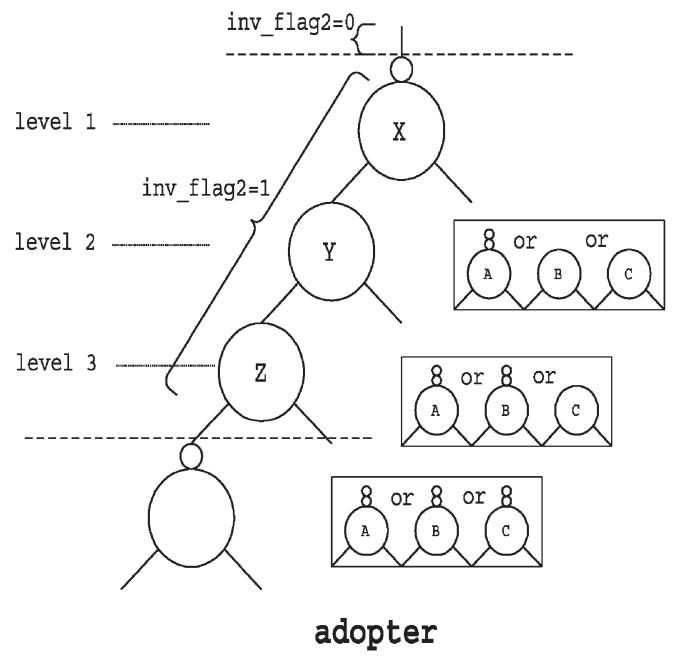

F2

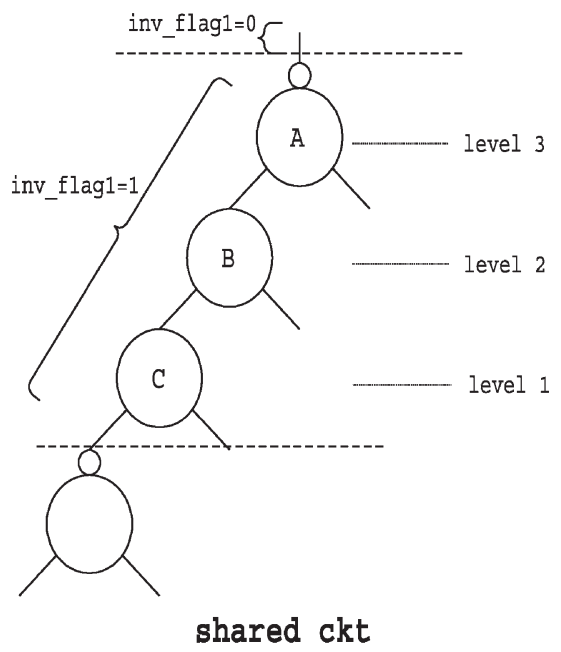

FIGURE 7 Insertion of inverters along 2-to-1 multiplexer chains of two Boolean functions when function F2 shares the sub-functions with function F1.

( 3 is a good choice according to our experiments). Thus, in our pass-transistor-based logic synthesizer, only two types of cells are required: 2-to-1 multiplexers and p-latch inverters, compared to the hundreds of cells in the traditional CMOS cell library. Figure 6d shows the aspect ratio of the two types of cells for 0.6 um CMOS process technology. Our synthesizer can generate circuit layout for any combinational logic functions expressed in terms of sum-of-product.

\section{Inverter/buffer Insertion}

One of the main goals of our synthesizer is to minimize the number of the extra inverters inserted in order to reduce the hardware cost and the critical-path delay. Our synthesizer allows users to specify the number of cascaded pass-transistors between two neighboring inverters being added. According to our experiment, it is suggested that an inverter should be added at every three cascaded pass-transistors. It is easy to add inverters for the BDD representation of a single Boolean function. First, we add an inverter at the primary output node at the root of the BDD tree. Then starting from the root, we search downward every path of the BDD tree and add an inverter whenever the number of cascaded node equals to three. After adding an inverter for a node, we have to invert all the leaves (1's or 0's) covered by that node in order to maintain the correctness of the logic function. We use a flag named inv_flag to record the possible complement of the sub-functions covered by the added inverter.

The inverter insertion procedure becomes complicated for simultaneous synthesis of several Boolean functions with shared sub-functions for hardware reduction. If we use the above inverter insertion method for each individual Boolean function, it is possible that the shared circuits among these functions might have pass-transistor chains

TABLE I Rules of inserting inverters or buffers for two Boolean functions with shared circuits in Fig. 7

\begin{tabular}{|c|c|c|c|}
\hline F1 & $\mathrm{F} 2$ & Same inv_flag & Opposite inv_flag \\
\hline \multirow[t]{3}{*}{ Level $1(\mathrm{X})$} & Level 3 (A) & $\begin{array}{l}\text { Generate buffered output } \\
\text { Connect buffered output }\end{array}$ & Connect inverted output \\
\hline & Level 2 (B) & Direct connection & $\begin{array}{l}\text { Generate inverted output } \\
\text { Connect inverted output }\end{array}$ \\
\hline & Level 1 (C) & Direct connection & $\begin{array}{l}\text { Generate inverter output } \\
\text { Connect inverted output }\end{array}$ \\
\hline \multirow[t]{3}{*}{ Level $2(\mathrm{Y})$} & Level 3 (A) & $\begin{array}{l}\text { Generate buffered output } \\
\text { Connect buffered output }\end{array}$ & Connect inverted output \\
\hline & Level 2 (B) & $\begin{array}{l}\text { Generate buffered output } \\
\text { Connect buffered output }\end{array}$ & $\begin{array}{l}\text { Generate inverted output } \\
\text { Connect inverted output }\end{array}$ \\
\hline & Level $1(\mathrm{C})$ & Direct connection & $\begin{array}{l}\text { Generate inverted output } \\
\text { Connect inverted output }\end{array}$ \\
\hline \multirow[t]{3}{*}{ Level 3 (Z) } & Level 3 (A) & $\begin{array}{l}\text { Generate buffered output } \\
\text { Connect buffered output }\end{array}$ & Connect inverted output \\
\hline & Level 2 (B) & $\begin{array}{l}\text { Generate buffered output } \\
\text { Connect buffered output }\end{array}$ & $\begin{array}{l}\text { Generate inverted output } \\
\text { Connect inverted output }\end{array}$ \\
\hline & Level 1 (C) & Generate buffered output & Generate inverted output \\
\hline
\end{tabular}




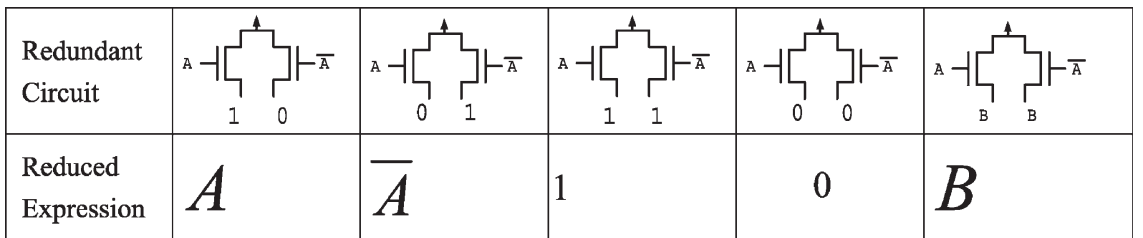

FIGURE 8 Possible hardware reduction for pass-transistor-based 2-to-1 multiplexers at the bottom level of BDD trees.

with more than three cascaded transistors. Thus, as shown in Fig. 7, after inverter insertion of the logic function F1 and F2 along the multiplexer chains, we need to perform extra operations when F1 uses the shared sub-function of F2. In order to avoid the situation of pass-transistor chains with more than three cascaded transistors during the multi-function sharing, we need to add a buffer (two sub-functions with same inv_flag) or an inverter (two sub-functions with opposite inv_flag) when node A, B or $\mathrm{C}$ in Boolean function F2 (called the shared circuit) is connected to one child of node $\mathrm{X}, \mathrm{Y}$ or $\mathrm{Z}$ in Boolean function F1 (called the adopter). Figure 7 and Table I show all the possible situation of function sharing and the necessary procedures to avoid multiplexer chains with more than three transistors. We observe that if the sum of function F1's level number (counting downward) and function F2's level number (counting upward) exceeds three, then either a buffer (or a inverter) is required depending on nodes X, Y, Z in F1 and nodes A, B, C in F2 that have the same (or opposite) inv_flags or not. Although we insert an inverter every three cascaded 2-to-1 multiplexers, the number of cascaded inverters is one of the input parameter specified by users.

After replacing each node of the BDD tree by a 2-to-1 multiplexer and inserting inverters along each multiplexer chain, we observe that the pass transistors that realize the 2-to-1 multiplexers at the bottom level of a BDD tree are redundant since the source/drain terminals of the MOS transistor are either VDD or GND. Figure 8 summarizes the possible hardware reduction and Fig. 9 shows an example of the synthesized circuits before and after the hardware reduction. Our synthesizer automatically eliminates these redundant circuits in order to reduce both the hardware cost and the critical path delay.

\section{Driving Capability Selection}

When a BDD tree is implemented using the multiplexers or inverters selected from a particular cell library consisting of different driving capability, as will be seen later, it is necessary to determine what kind of driving capability is needed. We use a method that calculates the importance value of each node in BDD trees. A node with larger importance value will be realized using multiplexers with larger driving capability.

The determination of importance value is illustrated in Fig. 10. In Fig. 10a, the fan-out (the number of upper branches) of node $\mathrm{A}$ is two while the fan-out of node $\mathrm{B}$ is one. Hence, node A has larger importance value since it has larger output load capacitance (connected to the input of two nodes. In Fig. 10b, the other branch of node C, i.e. the parent node of node $\mathrm{A}$, connects to a leaf of 0 or 1

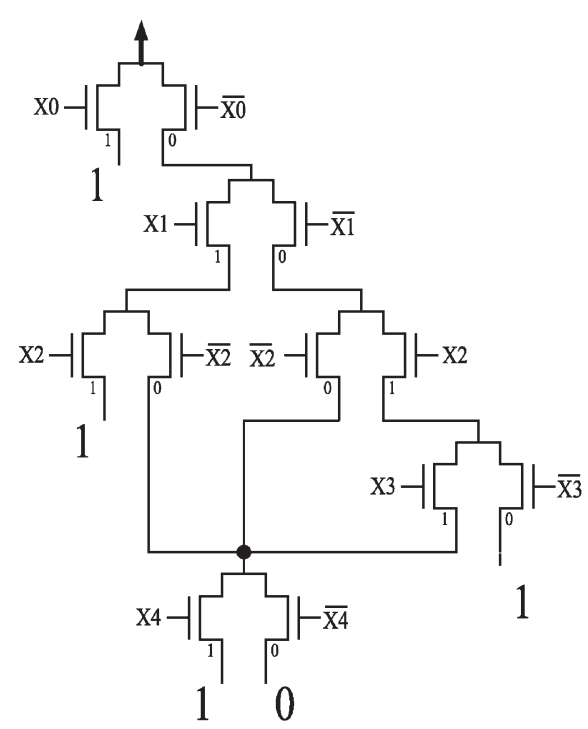

(a)

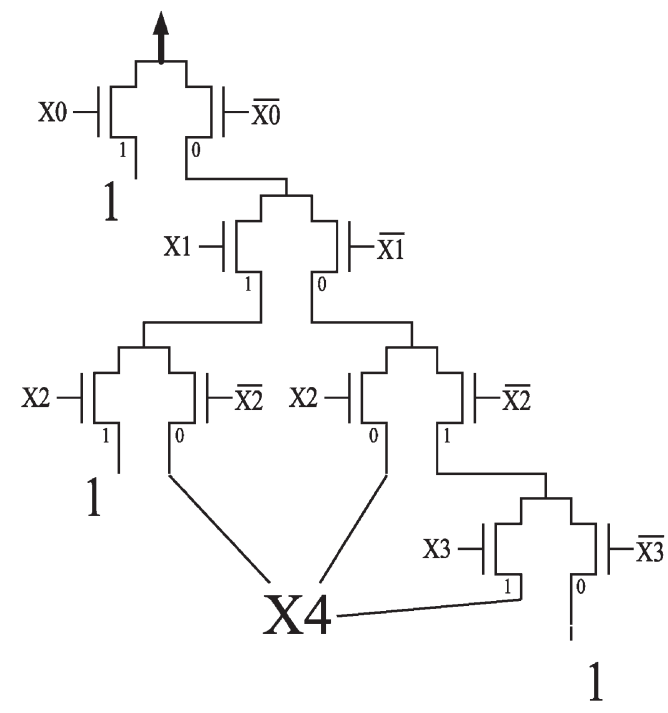

(b)

FIGURE 9 (a) A synthesized circuit with each node of a BDD tree realized by a pass-transistor-based 2-to-1 multiplexer and (b) a simplified circuit with the hardware reduction. 


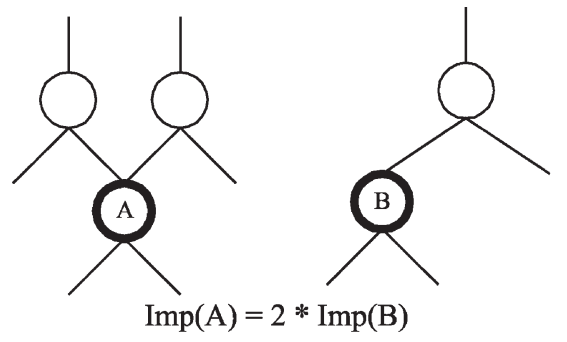

(a)

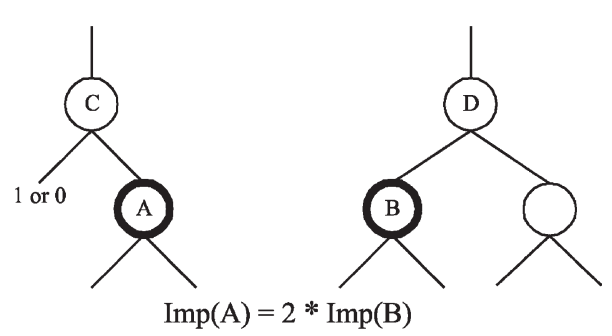

(b)

FIGURE 10 Determination of the importance value for a node. (a) Node A is more important than node B since A has larger output load. (b) Node A is more important than node $\mathrm{B}$ since $\mathrm{A}$ is more likely to be in the critical path.

while the other branch of node D (the parent node of node B) connects another non-trivial node (not a leaf). Hence, the probability of node B in the critical path is only about one half that of node $\mathrm{A}$. In this case, we assign node A the importance value twice larger than node B. Based on the above analysis, we define the importance value of node $\mathrm{A}$ as the ratio of the fan-out of node $\mathrm{A}$ and the non-trivial fan-in of its parent node, i.e.

$$
\operatorname{Imp}(\mathrm{A})=\frac{\text { fan }- \text { out of node } \mathrm{A}}{\text { non-trivial fan }- \text { in of A's parent node }} .
$$

Larger importance value represents the higher possibility of driving larger load at the more critical path. For example, in Fig. 10b, the fan-out of both node A and node $\mathrm{B}$ is one. However, the non-trivial fan-in of node A's parent node (node C) is 1 while the non-trivial fan-in of node B's parent node (node $\mathrm{D}$ ) is 2 . Thus, $\operatorname{Imp}(\mathrm{A})=1$ and $\operatorname{Imp}(B)=1 / 2$. We deduce that node $A$ needs larger driving capability since it is more important in the sense that it either drives larger load or has the higher probability of being at the critical path. Our logic synthesizer can automatically determine the proper driving capability of the multiplexers selected from the pass-transistor cell library based on the analysis of importance value for each node in the BDD representation of any logic function.

\section{Synthesis Based on 4-to-1 Multiplexers}

If the Boolean functions consist of a large number of input Boolean variables, the height (the maximum number of vertically cascaded nodes in the BBD) could be very large. Since each node in the BBD corresponding to a 2-to-1 multiplexer, the synthesized circuit could be very slow due to the long multiplexer chains. For example, in Fig. 11a, the implementation of the BDD tree of height 20 has a critical path going through 20 2-to-1 multiplexers plus 8 inserted inverters. Instead if we merge two BDD nodes into a new quadruple node, then the height of the new diagram reduces by half to 10 , as shown in Fig. 11b. In this case, each quadruple node is realized by a 4-to-1 multiplexer, realized by two 2-to-1 multiplexers as shown in Fig. 12a, with four different control signals generated from a selection circuit consisting of four 2-to-1 multiplexers and four inverters, as shown in Fig. 12b. Note that the path of the selection circuit lies at the horizontal direction while the delay path of the multiplexer-chain goes vertically. Thus, the overall critical path of the implementation based on 4-to-1 multiplexers contains only 11 2-to- 1 multiplexers plus 5 inserted inverters, a great improvement in speed performance at the cost of more hardware. This concept can be generalized for using higher-order multiplexers such as 8-to-1 multiplexers or 16-to-1 multiplexers. We have to emphasize that all

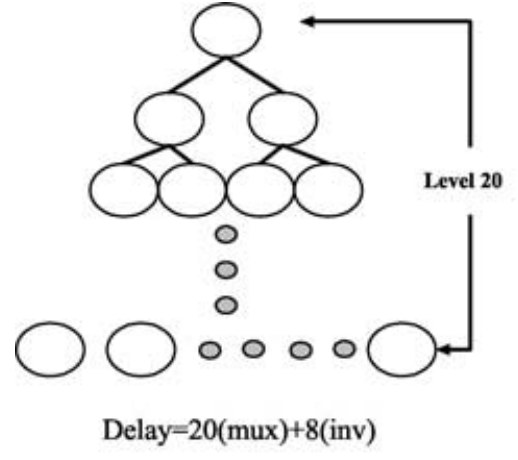

(a)

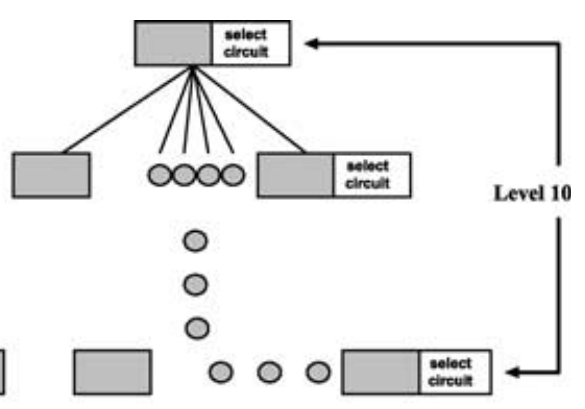

Delay $=11$ (mux) +6 (inv)

(b)

FIGURE 11 Synthesis of high performance circuits based on (a) 2-to-1 multiplexers and (b) 4-to-1 multiplexers. 


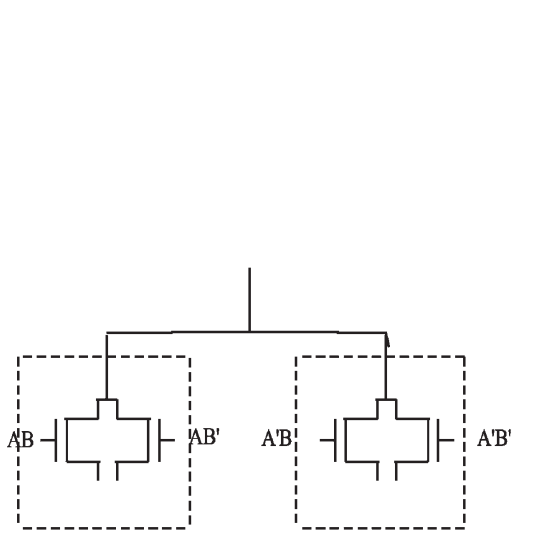

(a)

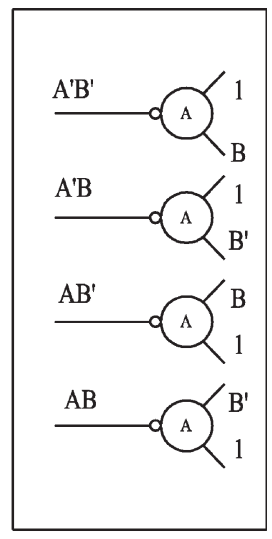

(b)
FIGURE 12 (a) 4-to-1 multiplexer constructed from two 2-to-1 multiplexers, and (b) the selection circuit to generate the control signals for the 4-to-1 multiplexer.

higher-order multiplexers can be realized from 2-to-1 multiplexers and inverters. In other words, the passtransistor cell library still contains only two types of cells. However, we found out that the hardware cost increases dramatically when using multiplexers with order larger than 4-to-1. Our synthesizer allows users to select implementation based on either 2-to-1 or 4-to-1 multiplexers, called high-density (HD) or high-performance (HP) synthesis, respectively.

\section{Parallelization for Speed Improvement}

In the previous subsection, we have shown one improvement method to speed-up the synthesized circuits by employing higher-order multiplexers in order to reduce the critical path delay. In fact, the delay can be further reduced through a parallelization technique by partitioning the critical path into several sections and parallelizing the logic evaluation of all sections. Figure 13a shows a synthesized circuit based on 4-to-1 multiplexers where we partition the critical path into four parts, named P1, P2, P3 and $\mathrm{P} 4$, by selecting three partition nodes $\mathrm{n} 1, \mathrm{n} 2$, and $\mathrm{n} 3$ where one input branch of each node is a leaf. The output of $\mathrm{P} 4$ is connected to one input branch of $\mathrm{n} 3$ and the output of $n 3$ is fed into P3. Thus, the output of P3 must wait for the output of $n 3$, which in turns depends on the outcome of $\mathrm{P} 4$. Since the output of $\mathrm{n} 3$ is either 1 or 0 , we can have two copies of P3, called P3_1 and P3_0 that execute concurrently with input from $\mathrm{n} 3$ assumed to be 1 and 0 , respectively. The correct result is selected using a new node, named $3^{\prime}$, with the control signal from the output of node n3. Similar duplications and concurrent operations can be applied to P2 and P1, as shown in Fig. 13b. Let TP1, TP2, TP3, and TP3 be the delays through P1, P2, P3, and $\mathrm{P} 4$, respectively. Compared to the original circuit of non-parallel version in Fig. 13a, the delay has been significantly reduced from around TP1 + TP $2+$ TP $3+$
TABLE II Speed and area comparison of various synthesized 8-bit carry-look-ahead (CLA8) adders

\begin{tabular}{lcc}
\hline CLA8 & Delay $(\mathrm{ns})$ & Transistor count \\
\hline CMOS & 5.4 & 625 \\
CPL & 5.9 & 637 \\
DPL & 6.6 & 912 \\
LEAP-like & 9.2 & 414 \\
HD & 6.4 & 402 \\
HD/P & 5.1 & 505 \\
HP & 5.7 & 547 \\
HP/P & 4.2 & 607 \\
\hline
\end{tabular}

TP4 to $\max (\mathrm{TP} 1, \mathrm{TP} 2, \mathrm{TP} 3, \mathrm{TP} 4)$. Our synthesizer is able to select the partition nodes in order to achieve better speed performance.

\section{EXPERIMENT RESULTS}

Figure 14 shows the flow chart of our logic/circuit synthesizer. There are only two types of cells in our passtransistor library: inverter, and 2-to-1 multiplexer. Each type of cell has four different kinds of driving capability. Our synthesizer generates for a group of input Boolean functions the transistor-level netlists with performance of either high speed (based on 4-to-1 multiplexers) or high density (based on 2-to-1 multiplexers), both with parallel versions for further speed improvement. The delays of the synthesized netlists are measured by HSPICE. Performance of synthesized circuits based on other types of cell library, such as conventional CMOS cell library, CPL, DPL or LEAP cell library, is also listed for comparison.

Table II compares several synthesized 8-bit carry-lookahead adders with different types of cells. First, we input a group of Boolean functions that produces the generate signals $\left(G_{i}\right)$ and the propagate signals $\left(P_{i}\right)$ in terms if the initial input bits $a_{i}, b_{i}$ and $c_{0}$. Then, the carry-out signals $\left(c_{i}\right)$ and the sum signals $\left(s_{i}\right)$ are expressed as Boolean functions of $G_{i}$ and $P_{i}$. Our synthesizer will consider hardware sharing of these Boolean functions using the method discussed in "Construction of BDD trees". The Synopsys synthesis results based on CMOS NOR and NAND gates are denoted by CMOS. CPL and DPL denote the implementations using CPL and DPL circuits, respectively. LEAP assimilates the original LEAP logic

TABLE III Speed and area comparison of various synthesized 64-bit carry-look-ahead adders constructed from two levels of CLA8 blocks

\begin{tabular}{lcc}
\hline CLA64 & Delay (ns) & Transistor count \\
\hline CMOS & 9.2 & 6683 \\
CPL & 9.4 & 6944 \\
DPL & 11.9 & 9896 \\
LEAP-like & 15.9 & 3961 \\
HD & 11.6 & 3925 \\
HD/P & 7.2 & 5073 \\
HP & 8.9 & 6038 \\
HP/P & 5.5 & 6351 \\
\hline
\end{tabular}




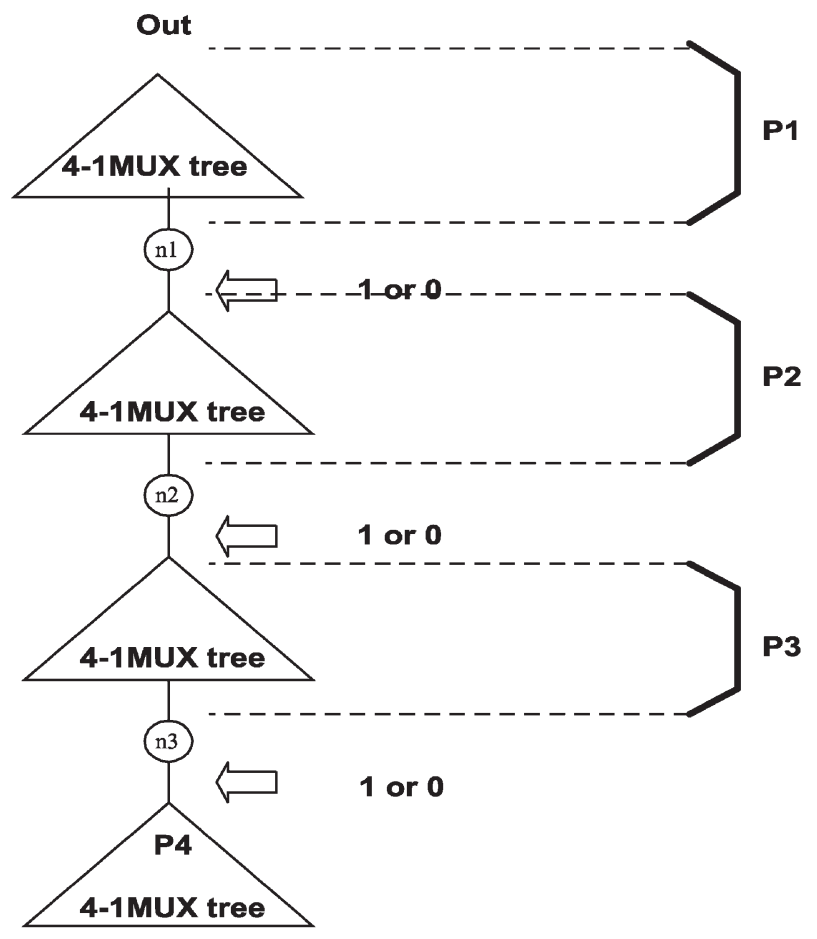

(a)

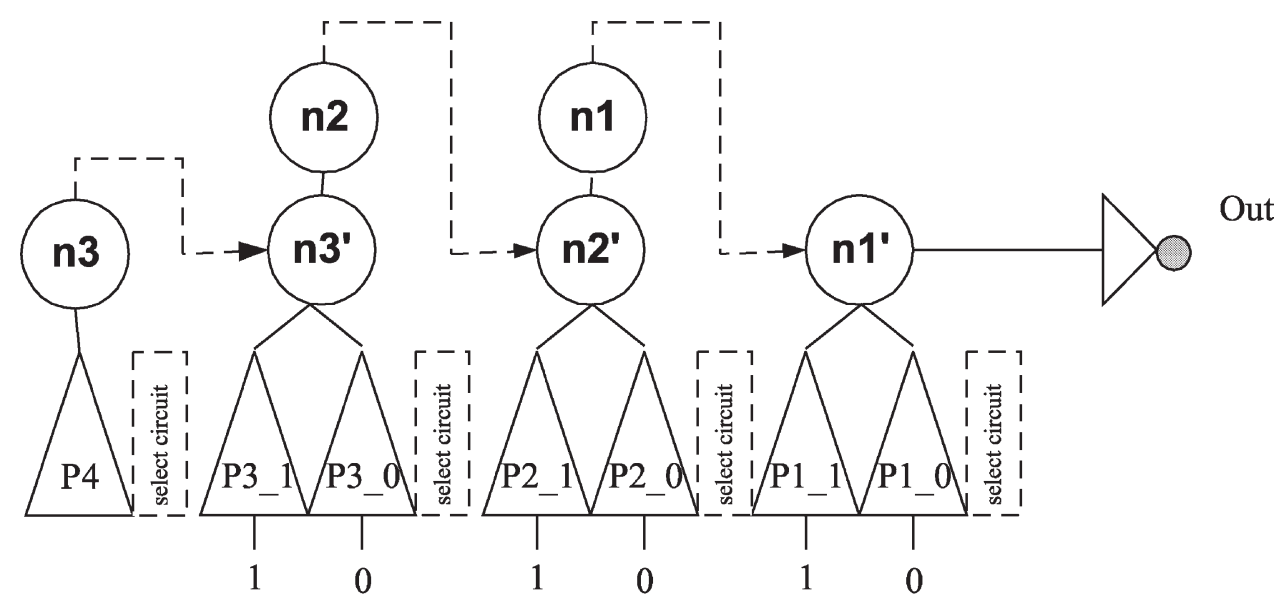

(b)

FIGURE 13 (a) Partitioning of a MUX-based logic, and (b) parallelization of the logic circuit for speed improvement.

with at most two cascaded multiplexers between the neighboring inverters inserted. HD is our synthesis results based on 2-to-1 multiplexers while HP are the implementations using 4-to-1 multiplexers to reduce the critical path (as discussed in "Implementation using passtransistor logic cell library"). HD/P and HP/P are the parallel versions of $\mathrm{HD}$ and $\mathrm{HP}$, respectively. We observe that our synthesis results (HD, HD/P, HP, HP/P) have better speed and/or area performance.

Table III lists the performance information of different synthesis results for a 64-bit CLA constructed from two levels of the 8-bit CLA blocks. Again, our HD, HP, HD/P, and HP/P implementations have either better area and/or speed performance compared with the original LEAP circuits or the conventional CMOS circuits in this example of a big fast adder design.

\section{CONCLUSIONS}

We presented a new top-down cell-based design method based on pass-transistor logic with only two types of cells: 


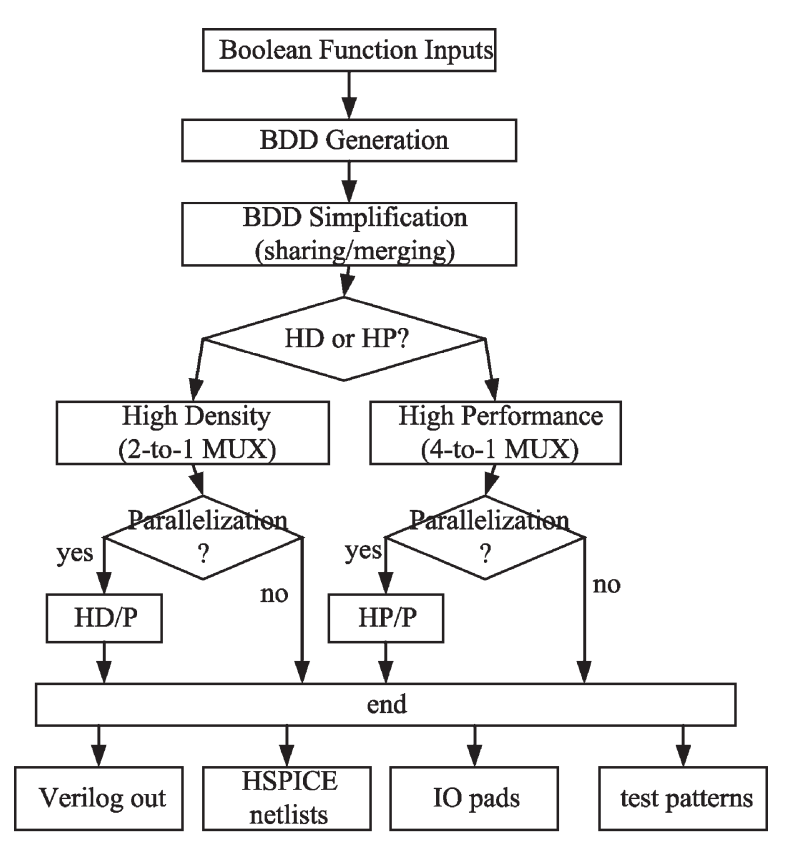

FIGURE 14 Flow chart of the logic/circuit synthesizer based on passtransistor cell library with only two types of cells: 2-to-1 multiplexer and inverter.

multiplexers and inverters, in the cell library. The corresponding logic/circuit synthesis tool using BDD tree construction is also developed which takes advantages of the characteristics of pass-transistor logic to minimize the delay. Further speed improvement can be achieved using higher-order multiplexers and/or a parallelization technique. Currently we are working on the logic synthesis of low-power circuits by considering the probability of switch activity associated with each node in the BDD trees. In addition, the transistor sizing of all the passtransistor can be done automatically for better driving capability selection.

\section{References}

[1] Yano, K., Yamanaka, T., Nishida, T., Saito, M., Shimohigashi, K. and Shimizu, A. (1990) "A 3.8-ns CMOS $16 \times 16-b$ multiplier using complementary pass-transistor logic", IEEE Journal of Solid-State Circuits 25(2), 388-395.

[2] Suzuki, M., Ohkubo, N., Shinbo, T., Yamanaka, T., Shimizu, A., Sasaki, K. and Nakagome, Y. (1993) "A 1.5-ns 32-b CMOS ALU in double pass-transistor logic", IEEE Journal of Solid-State Circuits 28(11), 1145-1150.

[3] Parameswar, A., Hara, H. and Sakurai, T. (1996) "A swing restored pass-transistor logic-based multiply and accumulate circuit for multimedia applications", IEEE Journal of Solid-State Circuits 31(6), 804-809.

[4] Lai, F. and Hwang, W. (1997) "Design and implementation of differential cascode voltage switch with pass-gate (DCVSPG) logic for high-performance digital systems", IEEE Journal of Solid-State Circuits, 563-573.

[5] Yano, K., Sasaki, Y., Rikino, K. and Seki, K. (1996) “Top-down passtransistor logic design", IEEE Journal of Solid-State Circuits 31(6), $792-803$.

[6] Akers, S.B. (1978) "Binary decision diagram", IEEE Transactions in Computers C-27, 509-516.

Shen-Fu Hsiao received his B.S. degree in Electrical Engineering from National Taiwan University, Taiwan in 1985, M.S. degree in Electronics from National ChiaoTung University, Taiwan in 1987, and the Ph.D. in Electrical Engineering from Yale University, USA in 1993. In 1993, he joined the Institute of Computer and Information Engineering at National Sun Yat-Sen University and is currently a Professor at Department of Computer Science and Engineering. His research interest includes computer arithmetic, VLSI design, and digital signal processing.

Jia-Siang Yeh received his M.S. degree from Institute of Computer and Information Engineering of National Sun Yat-Sen University, Taiwan in 1998. He is currently in obligatory military service.

Da-Yen Chen received his M.S. degree from Institute of Computer and Information Engineering of National Sun Yat-Sen University, Taiwan in 1999. He is currently in obligatory military service. 

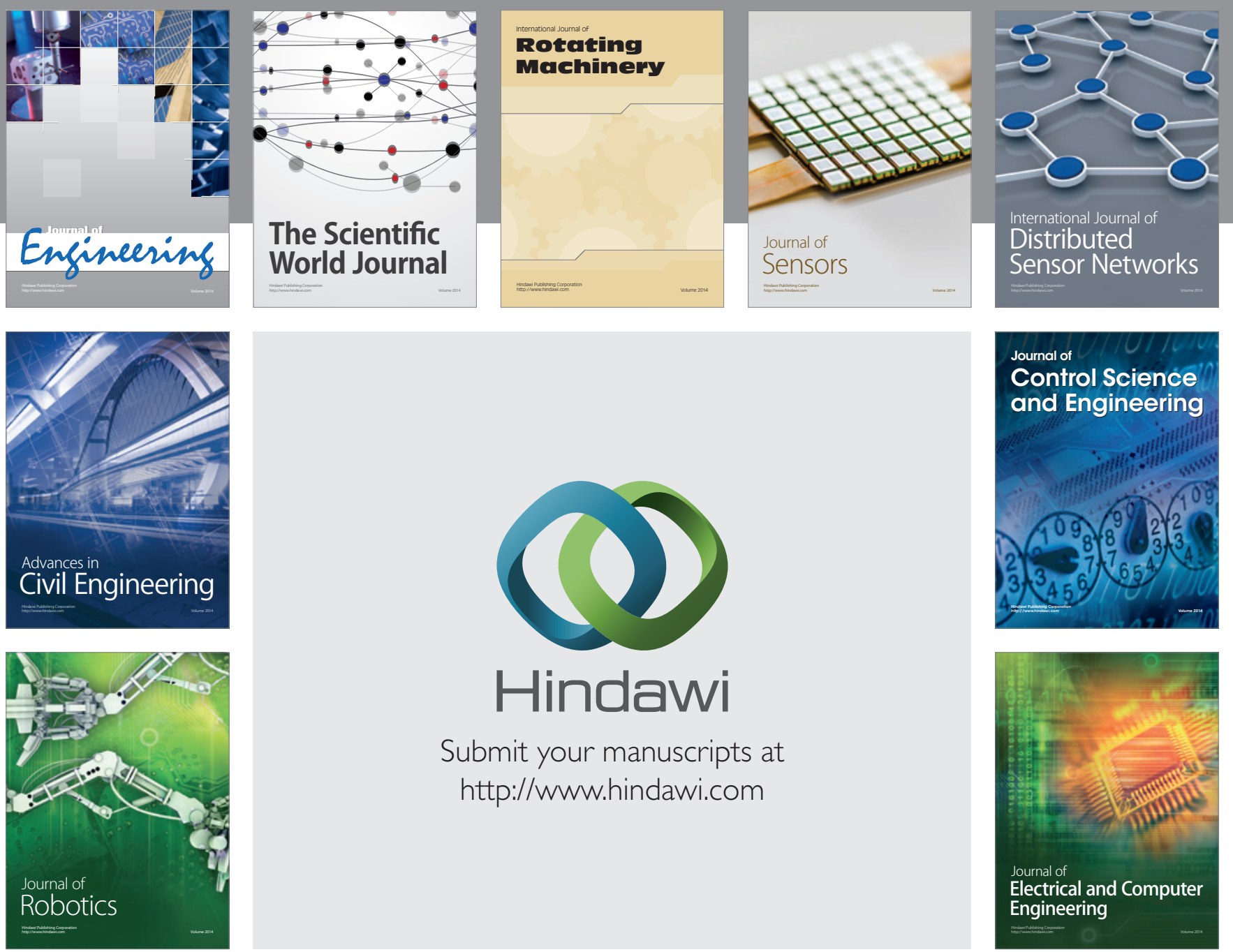

Submit your manuscripts at

http://www.hindawi.com
